Approved for public release; distribution is unlimited.

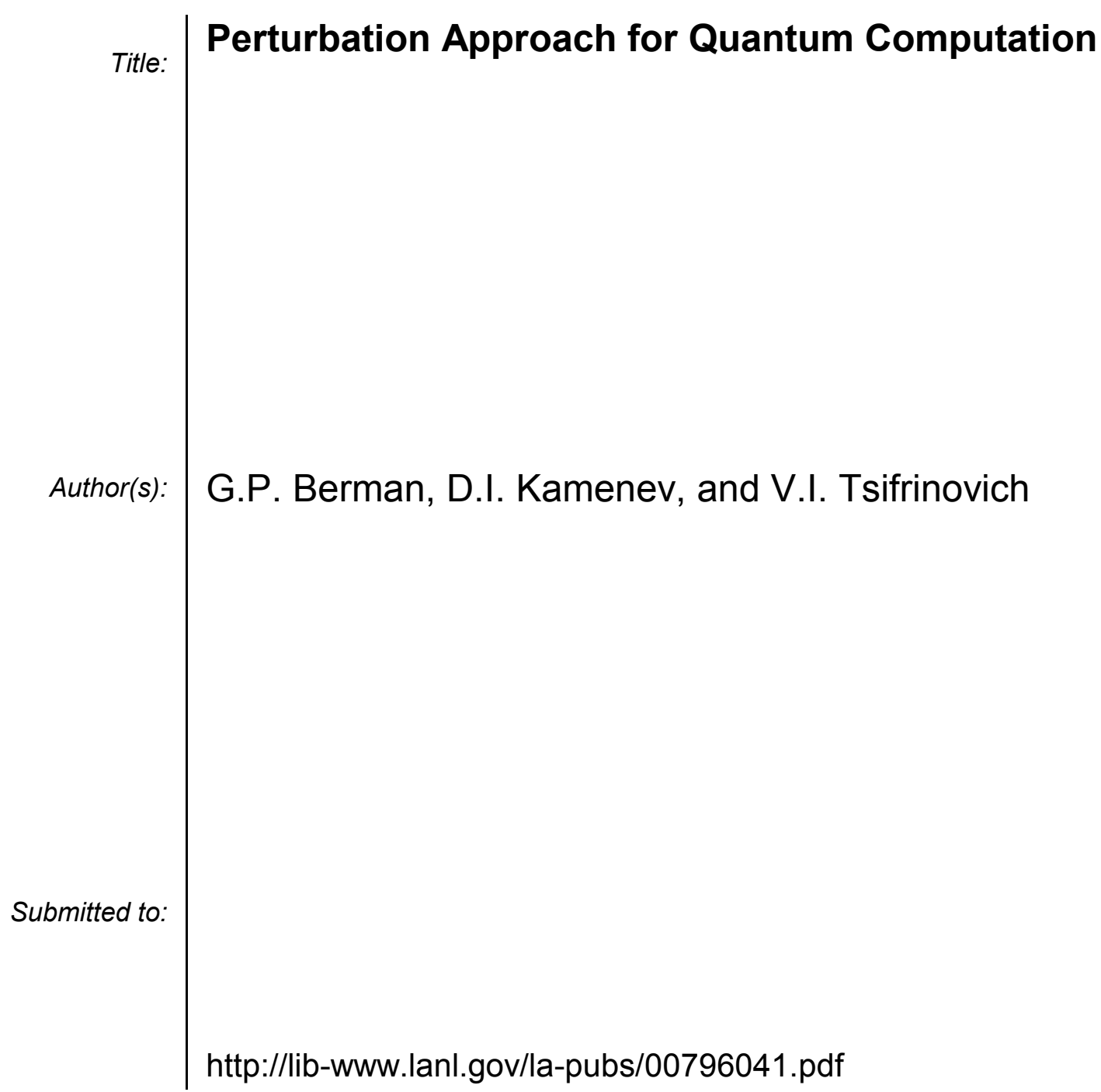

Los Alamos National Laboratory, an affirmative action/equal opportunity employer, is operated by the University of California for the U.S. Department of Energy under contract W-7405-ENG-36. By acceptance of this article, the publisher recognizes that the U.S. Government retains a nonexclusive, royaltyfree license to publish or reproduce the published form of this contribution, or to allow others to do so, for U.S. Government purposes. Los Alamos National Laboratory requests that the publisher identify this article as work performed under the auspices of the U.S. Department of Energy. Los Alamos National Laboratory strongly supports academic freedom and a researcher's right to publish; as an institution, however, the Laboratory does not endorse the viewpoint of a publication or guarantee its technical correctness. 


\title{
Perturbation Approach for Quantum Computation
}

\author{
G.P. Berman ${ }^{\dagger}$, D.I. Kamenev ${ }^{\dagger}$, and V.I. Tsifrinovich ${ }^{\ddagger}$ \\ $\dagger$ Theoretical Division and CNLS, Los Alamos National Laboratory, Los Alamos, \\ NM 87545 \\ ‡IDS Department, Polytechnic University, Six Metrotech Center, Brooklyn, \\ New York 11201
}

\begin{abstract}
We discuss how to simulate errors in the implementation of simple quantum logic operations in a nuclear spin quantum computer with many qubits, using radio-frequency pulses. We verify our perturbation approach using the exact solutions for relatively small $(L=10)$ number of qubits.
\end{abstract}

(C) 2001 Optical Society of America

OCIS codes: (200.3050) Information processing

We consider a chain of identical nuclear spins placed in an external nonuniform high magnetic field, $B(x)$, with a uniform gradient. The nuclear magnetic resonance (NMR) frequency for the $k$ th spin is $\omega_{k}=\gamma_{n} B_{k}$, where $\gamma_{n}$ is the nuclear gyromagnetic ratio and $B_{k}$ is the $z$-component of the magnetic field at the location of the $k$ th spin. The gradient of the magnetic field (in the direction of the chain, $x$ ) provides a shift of $\omega_{k}$ by the value $\delta \omega$ between the neighboring spins. (For the physical parameters see Ref. [1].)

The Hamiltonian of the spin chain in an external radio-frequency (rf) field is,

$H_{n}=-\sum_{k=0}^{L-1} \omega_{k} I_{k}^{z}-2 J \sum_{k=0}^{L-1} I_{k}^{z} I_{k+1}^{z}-\left(\Omega_{n} / 2\right) \sum_{k=0}^{L-1}\left[I_{k}^{-} \exp \left(-i\left(\nu_{n} t+\varphi_{n}\right)\right)+I_{k}^{+} \exp \left(i\left(\nu_{n} t+\varphi_{n}\right)\right)\right]=H_{0}+V_{n}(t)$,

where $J$ is the Ising interaction constant and $I_{k}^{z}$ is the operator of the $z$-component of spin $1 / 2, \Omega_{n}, \nu_{n}$ and $\varphi_{n}$ are the Rabi frequency, the frequency and the phase of the $n$th pulse, $I_{k}^{ \pm}=I_{k}^{x} \pm I_{k}^{y}$.

In this paper we estimate errors in creation of the entangled state from the ground state by applying a single $\pi / 2$ pulse and a sequence of $\pi$ pulses in the system described by the Hamiltonian (1). First, we describe schematically the protocol. The first $\pi / 2$ pulse creates a superposition of two states with equal probabilities, $|00 \ldots 00\rangle \rightarrow(1 / \sqrt{2})(|00 \ldots 00\rangle+i|10 \ldots 00\rangle)$. Other pulses transform this state to the entangled state,

$$
U \frac{1}{\sqrt{2}}(|00 \ldots 00\rangle+i|10 \ldots 00\rangle)=\frac{1}{\sqrt{2}}\left(e^{i \varphi_{1}}\left(|00 \ldots 00\rangle+e^{i \varphi_{2}}|10 \ldots 01\rangle\right),\right.
$$

where $\varphi_{1}$ and $\varphi_{2}$ are known phases (see Ref. [1]). The operator $U$ realizes a particular case of the well-known control-NOT gate. It has the following properties:

$$
\begin{gathered}
U \frac{1}{\sqrt{2}}\left|0_{L-1} 0_{L-2} \ldots 0_{1} 0_{0}\right\rangle=\frac{1}{\sqrt{2}} e^{i \varphi_{1}}\left|0_{L-1} 0_{L-2} \ldots 0_{1} 0_{0}\right\rangle, \\
U \frac{1}{\sqrt{2}}\left|1_{L-1} 0_{L-2} \ldots 0_{1} 0_{0}\right\rangle=\frac{1}{\sqrt{2}} e^{i\left(\varphi_{2}-\frac{\pi}{2}\right)}\left|1_{L-1} 0_{L-2} \ldots 0_{1} 1_{0}\right\rangle .
\end{gathered}
$$

To accomplish the operation (4) in the system described by the Hamiltonian (1), we choose a sequence of $\pi$ pulses with resonant frequencies. If we apply the same protocol to the ground state (operation (3)) then with some probability the system will remain in this state because these pulses are non-resonant to this state with the detunings $\Delta_{n} \neq 0$. The non-resonant transitions have the probabilities [1],

$$
\varepsilon_{n}=\left(\Omega_{n} / \lambda_{n}\right)^{2} \sin ^{2}\left(\lambda_{n} \tau_{n} / 2\right),
$$

where $\lambda_{n}=\sqrt{\Delta_{n}^{2}+\Omega_{n}^{2}}, \tau_{n}$ is the duration of the $n$th pulse. The values of the detunings, and Rabi frequencies are the same for all pulses, $\Delta_{n}=\Delta=2 J, \Omega_{n}=\Omega$, except for the fourth pulse, where $\Delta_{4}=4 J$ and $\Omega_{4}=2 \Omega$. For all pulses the values of $\varepsilon_{n}$ are the same, $\varepsilon=\varepsilon_{n}$.

We represent the wave function in the form,

$$
\Psi(t)=\sum_{p} A_{p}(t)|p\rangle \exp \left(-i \chi_{p}^{(n)} t\right), \quad \chi_{p}^{(n)}=\sum_{k=0}^{L-1} \sigma_{k}^{p} \frac{\nu_{n}}{2},
$$


where $\sigma_{k}^{p}=1$ if the $k$ th spin of the state $p$ is in the position 1 and $\sigma_{k}^{p}=-1$ if the $k$ th spin is in the position 0 , $|p\rangle$ are the eigenfunctions of the Hamiltonian $H_{0}$. Consider the dynamics during one pulse with the frequency $\nu_{n}$. The Schrödinger equation for the coefficients $A_{p}(t)$ has the form:

$$
i \dot{A}_{p}(t)=\left(E_{p}-\chi_{p}^{(n)}\right) A_{p}(t)-\frac{\Omega}{2} \sum_{p^{\prime}} A_{p^{\prime}}(t),
$$

where the sum is taken over the states $\left|p^{\prime}\right\rangle$ connected by a single-spin transition with the state $|p\rangle$.

Under the condition $\Delta \ll \delta \omega$ the distance between the $p$ th and the $m$ th diagonal elements connected by the resonant or near-resonant transition is $\left|\mathcal{E}_{p}^{(n)}-\mathcal{E}_{m}^{(n)}\right|=\Delta_{n}^{p m}$, where $\mathcal{E}_{p}^{(n)}=E_{p}-\chi_{p}^{(n)}$. This distance is much less than the distances to other diagonal elements. In this case one can neglect the interaction of the $p$ th state with all states except for the state $|m\rangle$. In this approximation the Hamiltonian matrix $\mathcal{H}_{p p^{\prime}}^{(n)}$ breaks up into $N / 2$ approximately independent matrices $2 \times 2$, where $N=2^{L}$.

This matrix approach allows us to estimate errors in the logic gate (2) caused by near-resonant and nonresonant transitions. Suppose that initially the eigenstate $|p\rangle$ of the Hamiltonian $H_{0}$ is populated. We want to calculate the probability of transition to the state $|r\rangle$ with $\left|E_{p}-E_{r}\right| \sim \delta \omega$, where the states $E_{p}$ and $E_{r}$ are connected by a flip of $k^{\prime}$ th spin, whose NMR frequency differs by the value $\sim \delta \omega$ from the frequency of the resonant transition. Since the matrix elements are small, $\left|V_{p r}^{(n)}\right|=\Omega / 2 \ll\left|\mathcal{E}_{p}^{(n)}-\mathcal{E}_{r}^{(n)}\right| \sim \delta \omega$, the eigenfunction, $\psi_{q}$, of the Hamiltonian $\mathcal{H}^{(n)}$ can be written in the form

$$
\psi_{q}=|q\rangle+\sum_{q^{\prime}}^{\prime} \frac{V_{q q^{\prime}}^{(n)}}{\mathcal{E}_{q}^{(n)}-\mathcal{E}_{q^{\prime}}^{(n)}}\left|q^{\prime}\right\rangle
$$

where prime in the sum means that the term with $q^{\prime}=q$ is omitted; $V_{q q^{\prime}}^{(n)}=-\Omega / 2$ for the states $|q\rangle$ and $\left|q^{\prime}\right\rangle$ connected by a single-spin transition of the $k^{\prime}$ th spin and $V_{q q^{\prime}}^{(n)}=0$ for all other states.

The probability of non-resonant transition from the state $|q\rangle$ to the state $|p\rangle$ is

$$
P_{p q}^{(n)}=\left|\left\langle p \mid \psi_{q}\right\rangle\right|^{2}=\left(\frac{V_{p q}^{(n)}}{\mathcal{E}_{q}^{(n)}-\mathcal{E}_{p}^{(n)}}\right)^{2} \approx\left(\frac{\Omega_{n}}{2\left|k-k^{\prime}\right| \delta \omega}\right)^{2},
$$

where $\left|k-k^{\prime}\right|$ is the distance from the $k^{\prime}$ th spin (whose NMR frequency, $\omega_{k^{\prime}}$, is non-resonant) to the $k$ th spin with the resonant (or near-resonant) NMR frequency. The probability $\mu_{L-1}$ (here the subscript of $\mu$ stands for the number of the resonant spin) of generation of unwanted states by the first $\pi / 2$ pulse in the result of non-resonant transitions is

$$
\mu_{L-1}=\left(\frac{\Omega}{2 \delta \omega}\right)^{2} \sum_{k^{\prime}=0}^{L-2} \frac{1}{\left|L-1-k^{\prime}\right|^{2}} .
$$

After the first $\pi / 2$ pulse the probability of correct procedure in implementation of the logic gate is $P_{1}=$ $1-\mu_{L-1}$. The probability of correct implementation of the operation (2) by applying $2 L-2$ pulses is

$$
\begin{gathered}
P_{2 L-2}=\frac{1}{2}\left(1-\mu_{L-1}\right)\left(1-\mu_{L-2}-\varepsilon\right)\left(1-4 \mu_{L-2}-\varepsilon\right)\left(1-\mu_{0}-\varepsilon\right) \prod_{i=1}^{L-3}\left(1-\mu_{i}-\varepsilon\right)^{2}+ \\
\frac{1}{2}\left(1-\mu_{L-2}\right)\left(1-4 \mu_{L-2}\right) \prod_{i=0}^{L-3}\left(1-\mu_{i}\right)^{2},
\end{gathered}
$$

where the first term is provided by the operation (3), and the last term appears due to (4).

In Fig. 1 (a) we compare the total probability, $\mathcal{P} \equiv 1-P_{2 L-2}$, of generation of unwanted sates with the result of exact solution in the case when the probability of near-resonant transitions is negligibly small, i.e. when $\varepsilon=0$. From Fig. 1 (a) one can see that in this case the probability $\mathcal{P}$ decreases as $\delta \omega$ increases.

In the case when $\varepsilon$ is large, $\varepsilon \gg \mu$, and $\Delta \ll \delta \omega$, the probability of errors is mostly defined by the value of $\varepsilon$ and is almost independent of $\delta \omega$. In Fig. 1 (b) we plot the probability $\mathcal{P}$ as a function of $\Omega$. The value of $\delta \omega$ defines the values of minima $\mathcal{P}_{\min }$ on Fig. 1 (b): the larger is $\delta \omega$ the deeper are the minima in the plot in Fig. 1 (b). The values of different minima in Fig. 1 (b) define the contribution of non-resonant processes 

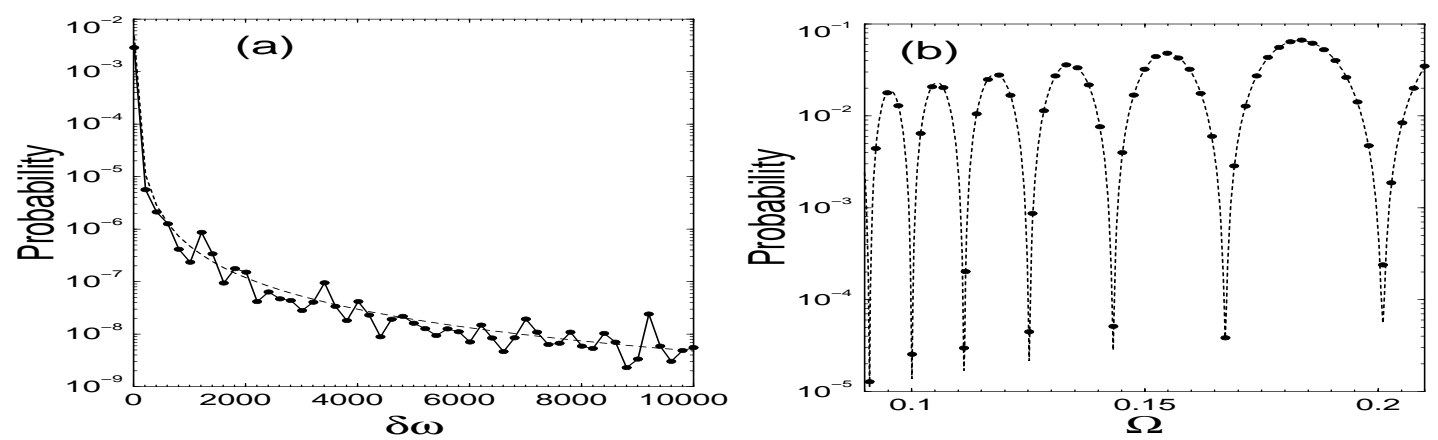

Fig. 1. (a) The probability, $\mathcal{P}$, of errors in implementation of the logic gate (2) at $\varepsilon=0$. Filled circles connected by the solid line - numerical results, dashed line - analytical estimate; the number of qubits $L=10, J=1, \Omega=2 J / \sqrt{\left(4 k^{2}-1\right)}, k=8$. (b) $\mathcal{P}$ as a function of $\Omega$. Filled circles - numerical results, dashed line - analytical estimate. $J=1, L=7, \delta \omega=100$.
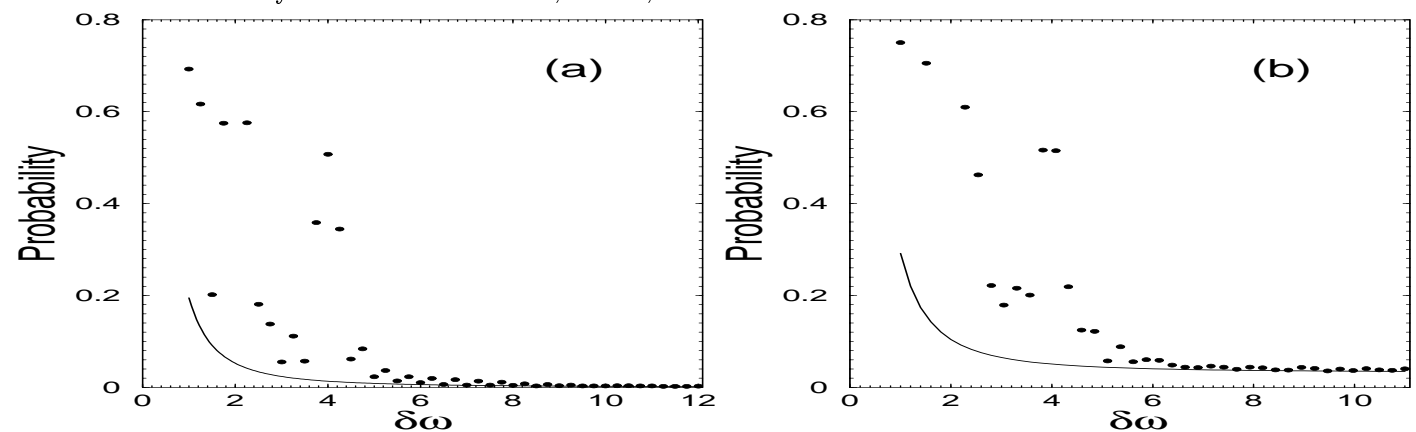

Fig. 2. The probability of errors in implementation of the logic gate $(2)$ as a function of $\delta \omega$ when the value of $\delta \omega$ is comparable with the value of the detuning, $\Delta$. (a) $\Omega=2 J / \sqrt{4 k^{2}-1}, \mathrm{k}=8(\varepsilon=0)$, (b) $\Omega=0.15$ $(\varepsilon=0.0039)$. Filled circles - numerical results, solid line - analytical estimate, $\mathcal{P} ; J=1, L=10$.

to the probability $\mathcal{P}$. Since the value of $\Omega$ in Fig. 1 (b) does not change significantly, the contribution of non-resonant processes to the probability of errors is approximately the same for all $\Omega$ and is equal to $\mathcal{P}_{\min }$. One can see that this contribution is negligibly small in comparison with the contribution of near-resonant processes (defined by $\varepsilon$ ) at all $\Omega$, except for the small regions of $\Omega$, where $\varepsilon$, given by Eq. (5), is minimal.

When the perturbation parameters of the problem, $\varepsilon$ and $\mu$, are small, by using Eq. (11) one can easily estimate the probability of errors in implementation of the logic gate (2) when the number of qubits in the spin chain is large (for example, for $L \sim 1000$ ). In this case the equation (11) is important for estimation of errors, since the exact solution of the problem requires diagonalization of the enormous matrices of the size $2^{L} \times 2^{L}$.

We should note that one more condition, except for $\varepsilon, \mu \ll 1$, must be satisfied for applicability of Eq. (11). The value of detuning, $\Delta$, should be small in comparison with the difference between NMR frequencies of the spins $\Delta \ll \delta \omega$. In Figs. 2 (a) and (b) we plot the probability, $\mathcal{P}$, as a function of $\delta \omega$ for small and large values of $\varepsilon$. One can see that our results are valid only in the case when $\Delta \ll \delta \omega$, in spite of the parameter $\Delta / \delta \omega$ does not appear explicitly in Eq. (11). From Fig. 2 (b) one can see that the probability of errors, $\mathcal{P}$, at $\varepsilon \gg \mu$ and at large $\delta \omega$ becomes relatively independent of $\delta \omega$. In this case the value of $\mathcal{P}$ is defined by $\varepsilon$ which, due to Eq. (5), does not depend on $\delta \omega$.

This work was supported by the Department of Energy (DOE) under contract W-7405-ENG-36, by the National Security Agency (NSA), and by the Advanced Research and Development Activity (ARDA).

\section{References}

1. G.P. Berman, G. D. Doolen, G. V. Lòpez, and V. I. Tsifrinovich, Phys. Rev. A 61, 2305 (2000).

2. G.P. Berman, G. D. Doolen, R. Mainieri, and V. I. Tsifrinovich, Introduction to Quantum Computers (World Scientific, Singapore, 1998). 